\title{
Algorithms for mathematical morphology
}

\author{
M. Van Droogenbroeck
}

October 8, 2002 


\section{Outline}

- Introduction

- Review of existing algorithms and discussion

- Description of two recent algorithms

- Comparison

- Granulometries 


\section{Introduction}

- Do we really need new algorithms for openings?

- Are existing algorithms not fast enough? 


\section{Introduction}

- Do we really need new algorithms for openings?

- Are existing algorithms not fast enough?

- Applications

- Industrial applications

- Label images

- Requirements

- Hardware or software?

- Memory requirements?

- Is speed the ultimate goal?

- Should the implementation be reusable? 


\section{Definitions and interpretations}

- The erosion $X \ominus B$ and dilation $X \oplus B$ of a set $X$ are defined as:

$$
\begin{aligned}
& X \ominus B=\bigcap_{b \in B} X_{-b} \\
& X \oplus B=\bigcup_{b \in B} X_{b}
\end{aligned}
$$

For a function $f$

$$
f \ominus B=\bigwedge_{b \in B} f_{-b} \quad f \oplus B=\bigvee_{b \in B} f_{b}
$$


- The opening

$$
X \circ B=(X \ominus B) \oplus B
$$

- Geometric interpretation:

$$
X=\bigcup\left\{B_{p} \mid B_{p} \subseteq X\right\}
$$




\section{Notations and conventions}

- Size of a structuring element: a set $B$ of size $n$, denoted $n B$, is usually defined as

$$
n B=\underbrace{B \oplus B \oplus \ldots \oplus B}_{n-1 \text { dilations }}
$$




\section{Notations and conventions}

- Size of a structuring element: a set $B$ of size $n$, denoted $n B$, is usually defined as

$$
n B=\underbrace{B \oplus B \oplus \ldots \oplus B}_{n-1 \text { dilations }}
$$

- Here we adopt a different convention!

- $n H$ is a $n$-pixels wide segment. 


\section{Notations and conventions}

- Size of a structuring element: a set $B$ of size $n$, denoted $n B$, is usually defined as

$$
n B=\underbrace{B \oplus B \oplus \ldots \oplus B}_{n-1 \text { dilations }}
$$

- Here we adopt a different convention!

- $n H$ is a $n$-pixels wide segment.

- $B$ is a square or a rectangle.

- $H$ is an horizontal segment and $V$ is a vertical segment

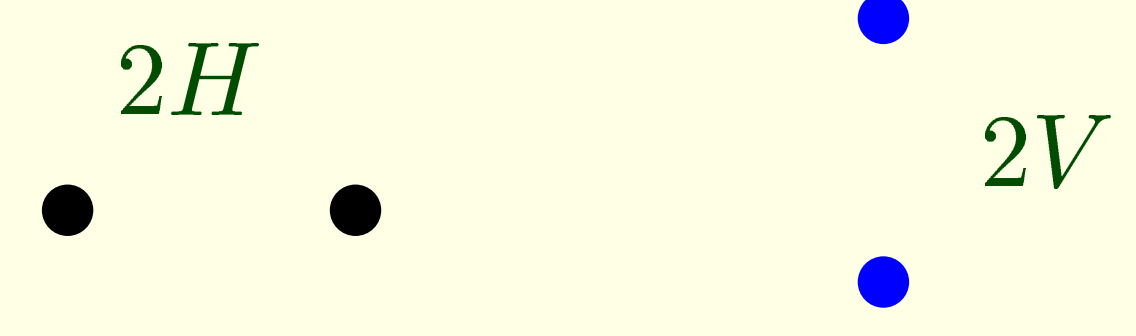




\section{Review of existing algorithms}

Problem statement:

- Suppose $B=n H \oplus m V$, we need to compute $X \circ(n H \oplus m V)$. 


\section{Review of existing algorithms}

Problem statement:

- Suppose $B=n H \oplus m V$, we need to compute $X \circ(n H \oplus m V)$.

Classification of different algorithms?

- Elementary implementations

- Histogram based

- VAN HERK's algorithm

- Algorithms dedicated to binary images

- Distance based algorithms

- Algorithms based on the geometric interpretation of openings 


\section{Elementary implementations}

1. Trivial implementation

(a) direct application of the definition $\Rightarrow$ complexity proportional to $n \times m$ 


\section{Elementary implementations}

1. Trivial implementation

(a) direct application of the definition $\Rightarrow$ complexity proportional to $n \times m$

2. Alternative: linear decomposition.

(a) based on the chain rule $X \ominus(n H \oplus m V)=(X \ominus n H) \ominus m V$

(b) results in $(((X \ominus n H) \ominus m V) \oplus n H) \oplus m V$.

(c) complexity proportional to $n+m$. 


\section{Elementary implementations}

1. Trivial implementation

(a) direct application of the definition $\Rightarrow$ complexity proportional to $n \times m$

2. Alternative: linear decomposition.

(a) based on the chain rule $X \ominus(n H \oplus m V)=(X \ominus n H) \ominus m V$

(b) results in $(((X \ominus n H) \ominus m V) \oplus n H) \oplus m V$.

(c) complexity proportional to $n+m$.

3. Other alternative: logarithmic decomposition

(a) Proposed by Pecht (1985) and VAN DEn BoomgaArd (1992)

(b) The logarithmic decomposition suppresses the redundancy inherent to recursive dilations with a unique convex structuring element. 


\section{Logarithmic decomposition}

For example, if $\partial(B)$ denotes the border of $B$,

$$
9 B=B \oplus \partial(B) \oplus \partial(B) \oplus \partial(2 B) \oplus \partial(4 B)
$$

(usual notation of size) 


\section{Logarithmic decomposition}

For example, if $\partial(B)$ denotes the border of $B$,

$$
9 B=B \oplus \partial(B) \oplus \partial(B) \oplus \partial(2 B) \oplus \partial(4 B)
$$

(usual notation of size)

\section{Theorem.}

$$
B \oplus B=B \oplus \partial(B)
$$

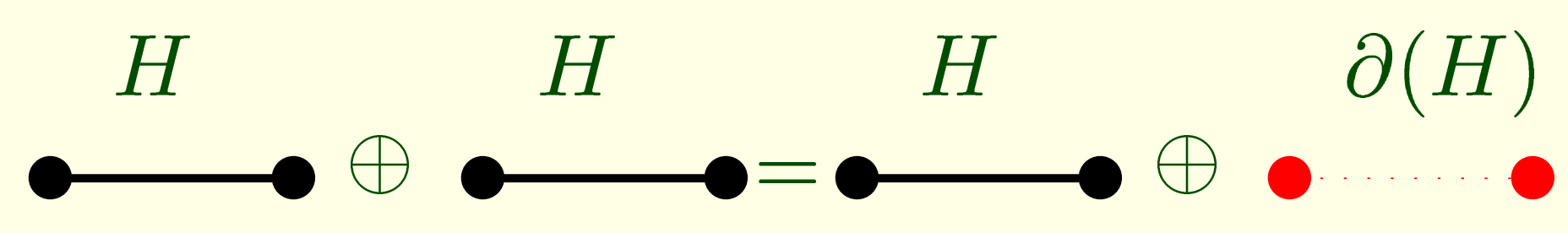




\section{Logarithmic decomposition}

For example, if $\partial(B)$ denotes the border of $B$,

$$
9 B=B \oplus \partial(B) \oplus \partial(B) \oplus \partial(2 B) \oplus \partial(4 B)
$$

(usual notation of size)

Theorem.

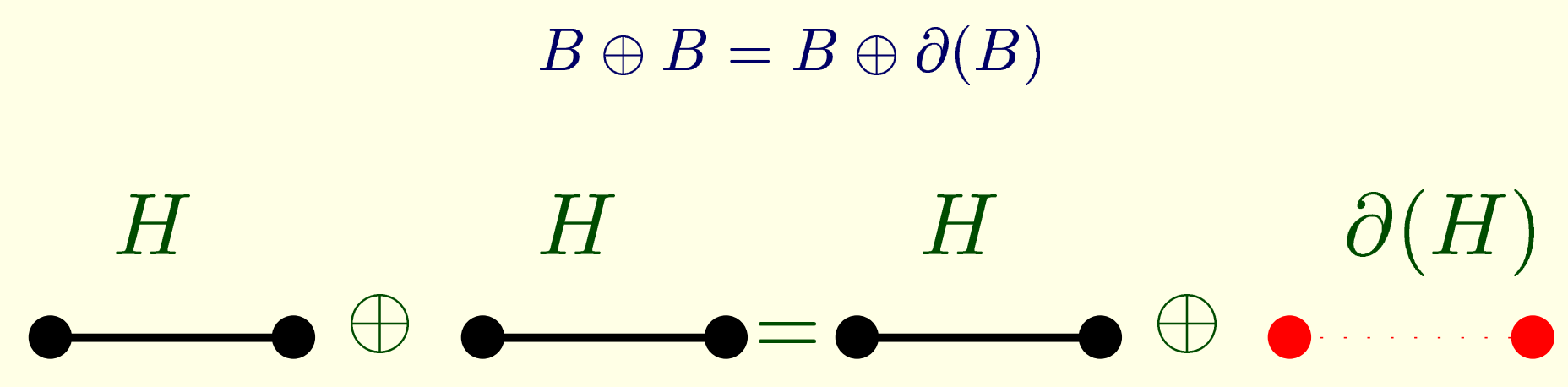

$\Rightarrow$ Other decompositions are possible:

$$
9 B=B \oplus \partial(B) \oplus \partial(B) \oplus \partial(3 B) \oplus \partial(3 B)
$$




\section{Histogram based algorithms (I)}

Original idea of HUANG et al. [1979]:

- a local histogram is computed in a sliding window.

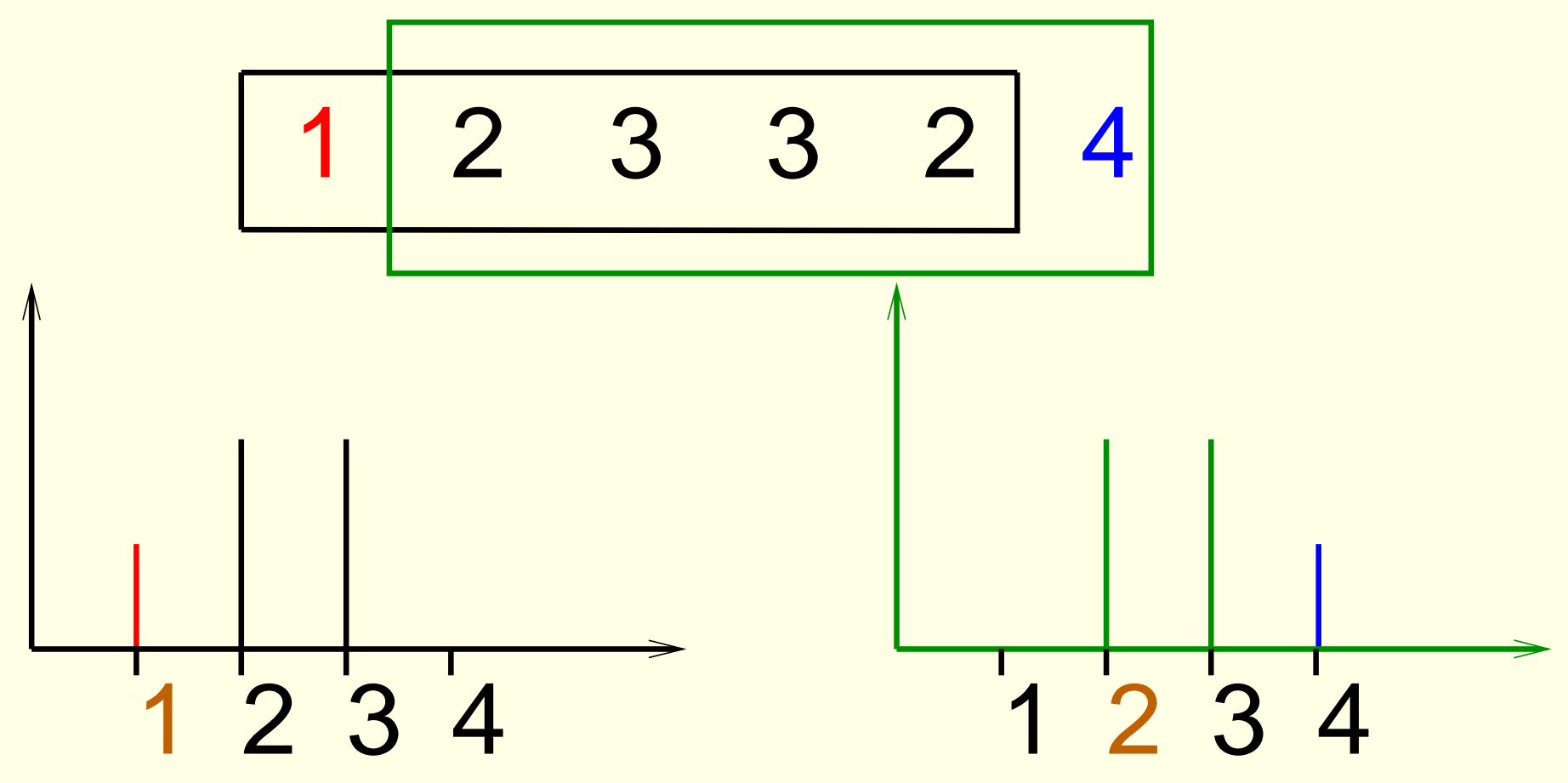

Figure 1: Illustration in the case of an erosion. 


\section{D structuring elements}
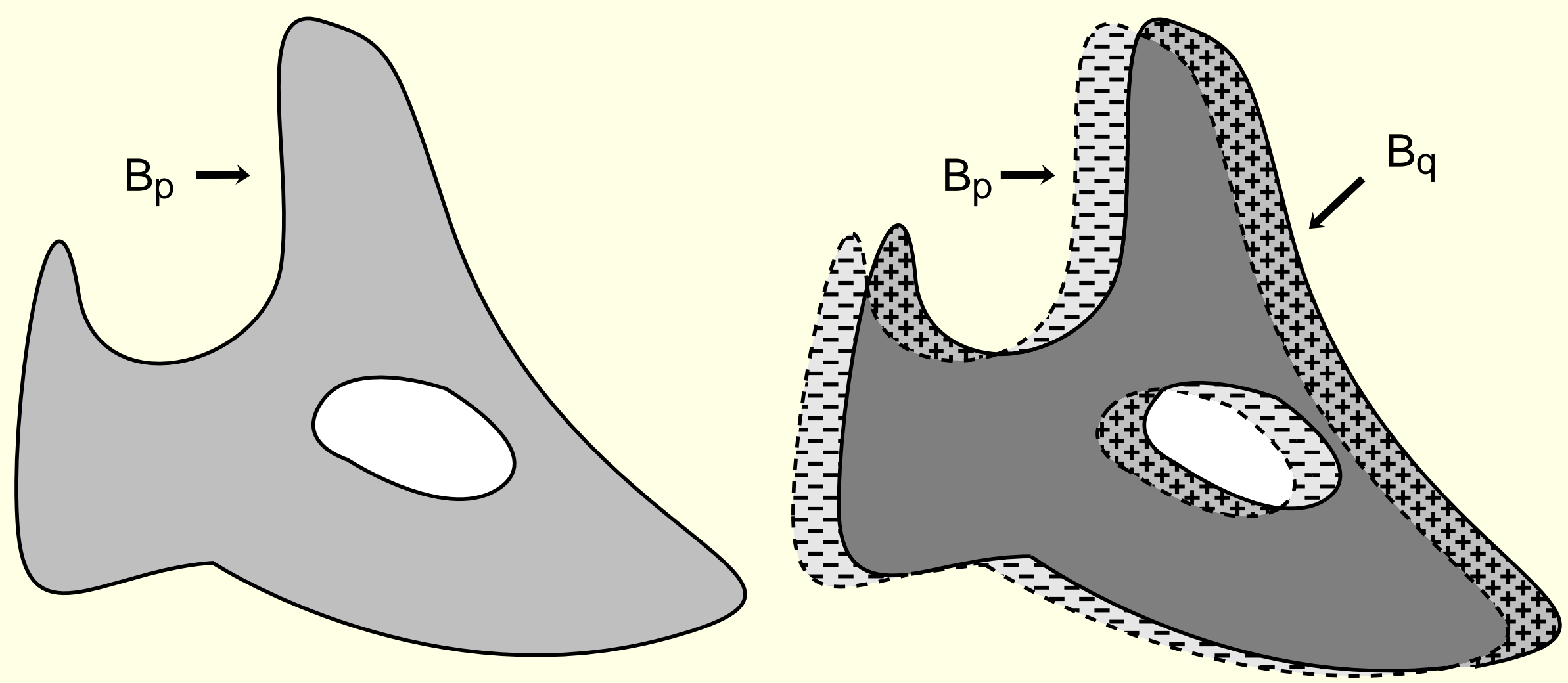

To be removed

To be added 

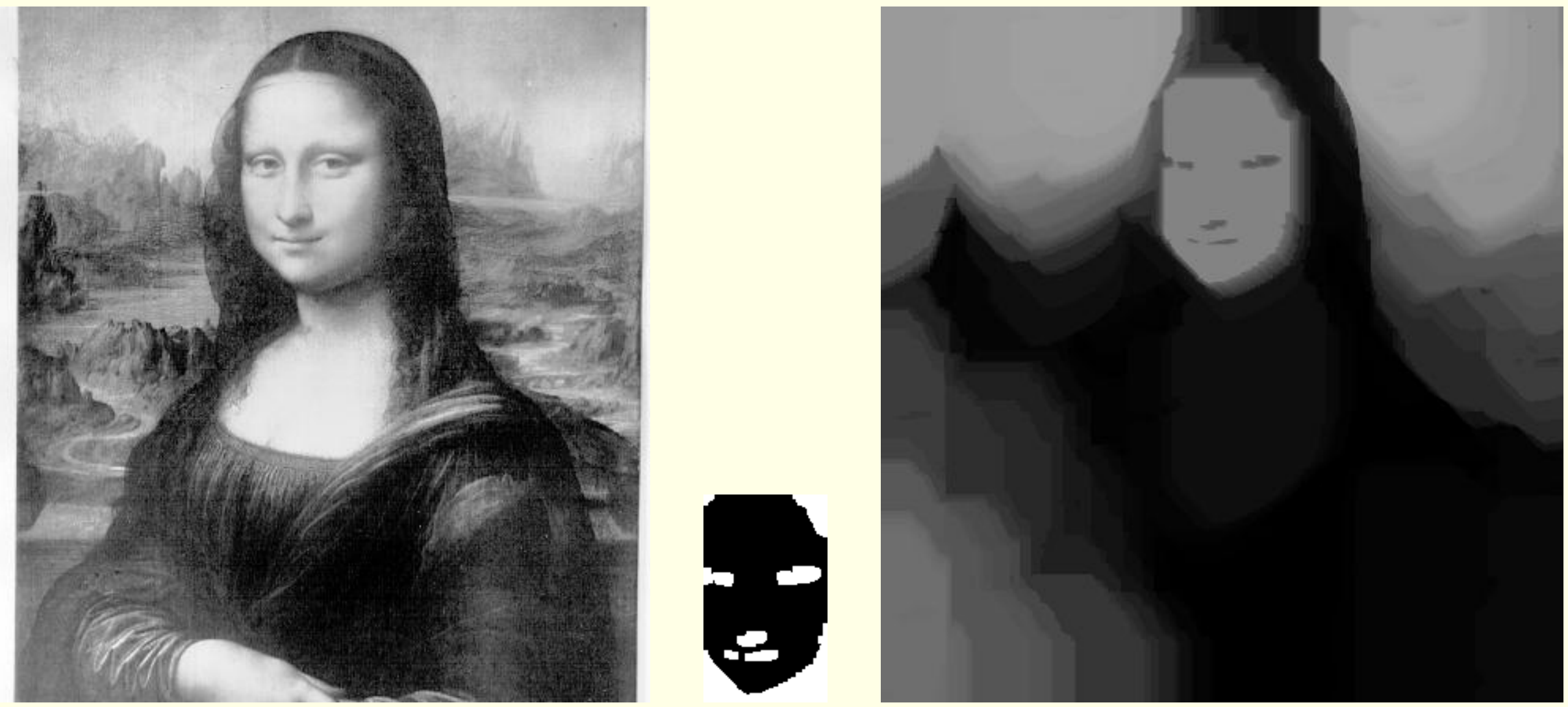


\section{Statistical properties of an erosion/dilation}

Paper by STEVENSON (1996)

\section{Notations}

Let $X_{1}, X_{2}, \ldots, X_{N}$ be random variables with a same distribution.

$f_{X}(x)$ is the PDF and $F_{X}(x)=P(X \leq x)$

We define: $Y=\min \left\{X_{1}, X_{2}, \ldots, X_{N}\right\}$ et $Z=\max \left\{X_{1}, X_{2}, \ldots, X_{N}\right\}$.

Then [Dilation]

$$
\begin{aligned}
F_{Z}(z)=P(Z \leq z) & =P\left(\max \left\{X_{1}, X_{2}, \ldots, X_{N}\right\} \leq z\right) \\
& =P\left(X_{1} \leq z, X_{2} \leq z, \ldots, X_{N} \leq z\right) \\
& =P\left(X_{1} \leq z\right) P\left(X_{2} \leq z\right) \ldots P\left(X_{N} \leq z\right) \\
& =\left(F_{X}(z)\right)^{N}
\end{aligned}
$$

and

$$
f_{Z}(z)=\frac{d F_{Z}(z)}{d z}=\frac{d\left(F_{X}(z)\right)^{N}}{d z}=N f_{X}(z)\left(F_{X}(z)\right)^{N-1}
$$


[Erosion]

$$
\begin{aligned}
F_{Y}(y)=P(Y \leq y) & =P\left(\min \left\{X_{1}, X_{2}, \ldots, X_{N}\right\} \leq y\right) \\
& =1-P\left(\min \left\{X_{1}, X_{2}, \ldots, X_{N}\right\}>y\right) \\
& =1-P\left(X_{1}>y\right) P\left(X_{2}>y\right) \ldots P\left(X_{N}>y\right) \\
& =1-\left(1-P\left(X_{1} \leq y\right)\right)^{N} \\
& =1-\left(1-F_{X}(y)\right)^{N}
\end{aligned}
$$




\section{What could we do when a $\mathrm{min} / \mathrm{max}$ value is lost?}

Solution: built a list of local maximums and keep them in memory.

DougLAS (1996). Running Max/Min calculation using a pruned ordered list. 


\section{Histogram based algorithms (II)}

In the case of a binary image:

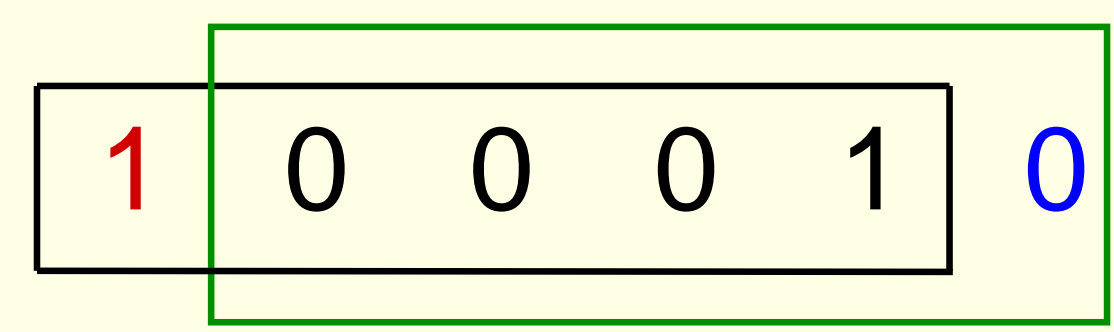

- only a single counter is needed 


\section{Histogram based algorithms (II)}

In the case of a binary image:

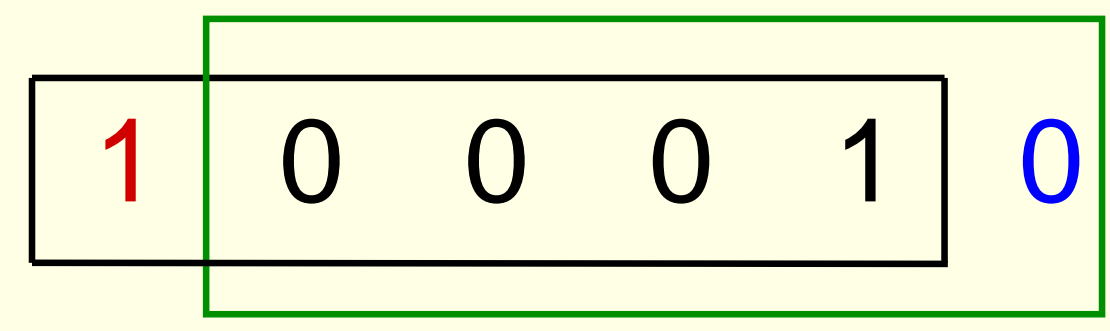

- only a single counter is needed

Properties:

- the computation time does not depend on the size of the structuring element

- the computation time depends on the image content

- applicable to arbitrary structuring elements 


\section{VAN HERK's algorithm (I)}

VAN HERK (1992) proposed a 1-D algorithm based on separability and a combination of block recursive series which are evaluated forwards and backwards.

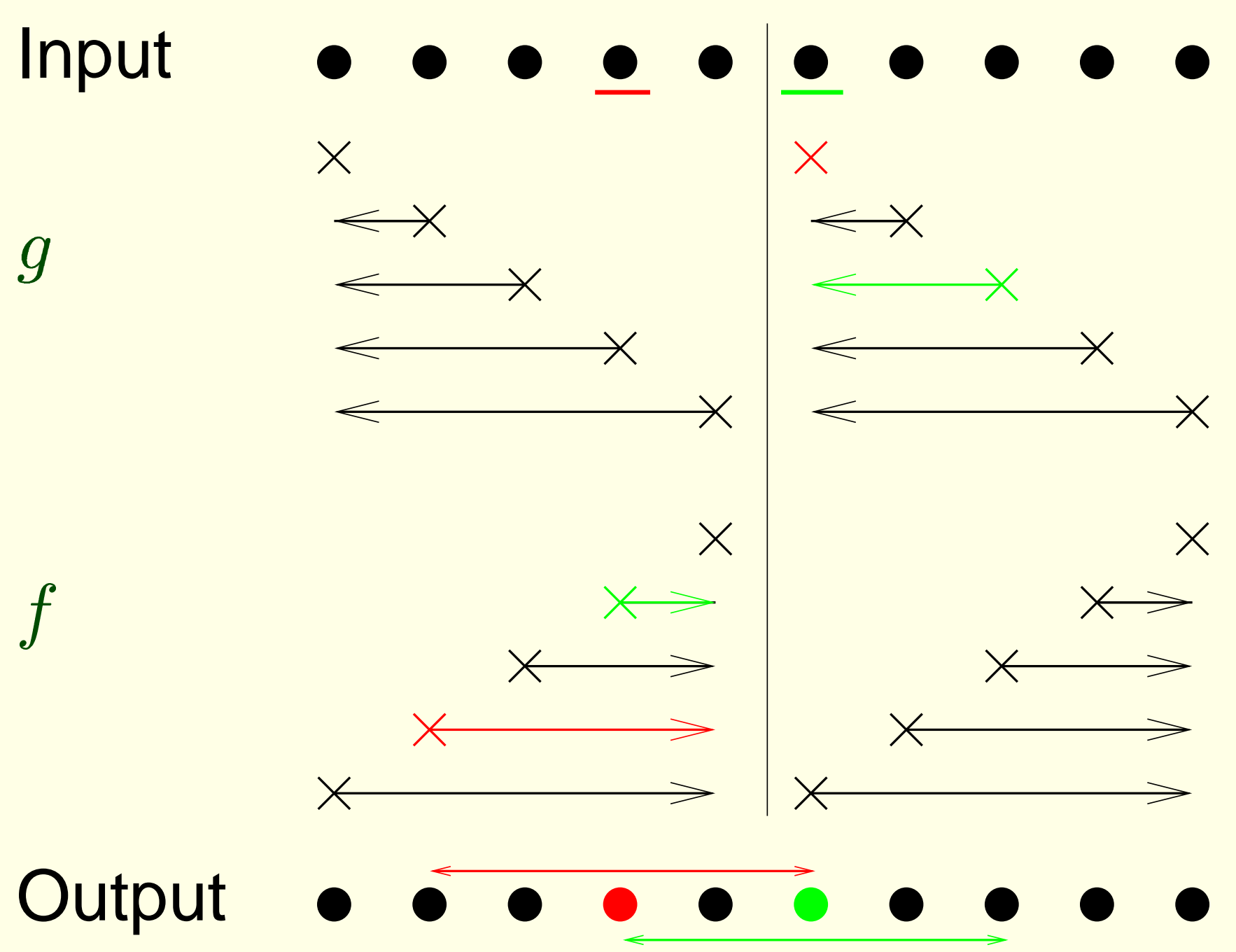




\section{VAN HERK's algorithm (II)}

\section{Properties:}

- 3 comparisons per pixel

- number of operations independent of the structuring element size

- but the structure is dependent of the structuring element size!

- applicable to binary morphology

Further improvements proposed by GIL and KIMMEL (ISMM 2000)

VAN HERK's algorithm will be used for benchmarking 


\section{Directional Morphological Filtering}

Problem statement: how should we handle an erosion on a Bresenham line? Answer: papers by JONES, SOILLE and TALBOT (2002) 


\section{Algorithms dedicated to binary morphology}

- Young et al. (1981) has proposed methods suited for hardware implementations

- VAN VLIET et al. (1988), SCHMITT (1989), and VINCENT (1991) provided methods which analyze the border of both $X$ and $B$. 


\section{Distance based algorithms}

LAY (1987) developed an algorithm based on the distance function.

$$
\begin{array}{cccccccccccccc}
X & 0 & 1 & 1 & 1 & 1 & 1 & 1 & 0 & 1 & 1 & 1 & 1 & 0 \\
\text { Distance } & 0 & 1 & 2 & 3 & 3 & 2 & 1 & 0 & 1 & 2 & 2 & 1 & 0 \\
X \ominus 5 H & 0 & 0 & 0 & 1 & 1 & 0 & 0 & 0 & 0 & 0 & 0 & 0 & 0
\end{array}
$$




\section{Algorithms based on the geometric interpretation of openings}

Propagation algorithm for binary openings with a rectangle
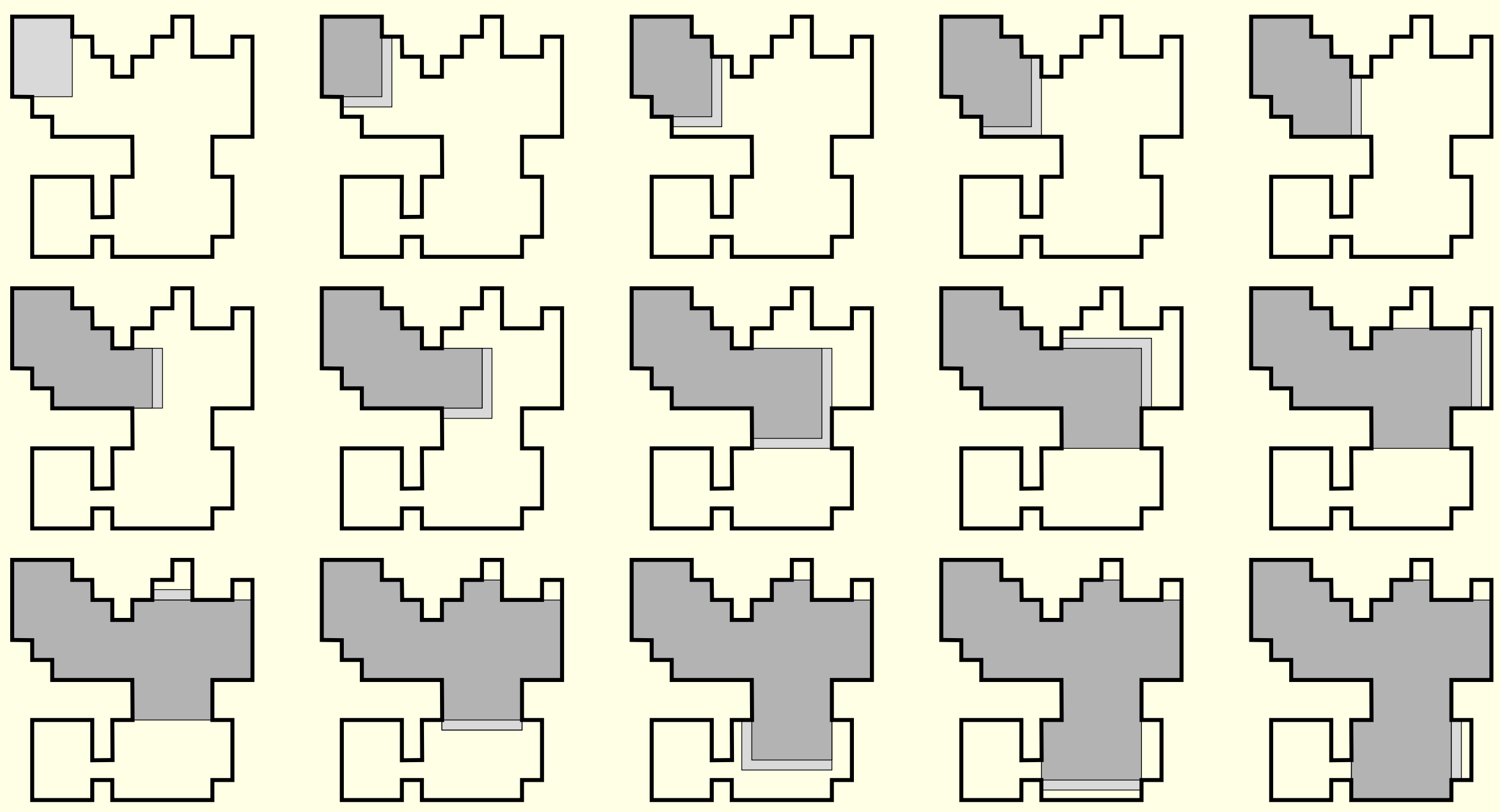


\section{$\underline{\text { Remark: }}$}

$$
(X \circ B) \ominus B=X \ominus B
$$

$\Rightarrow$ the opening provides the erosion! 


\section{Single pass opening algorithms}
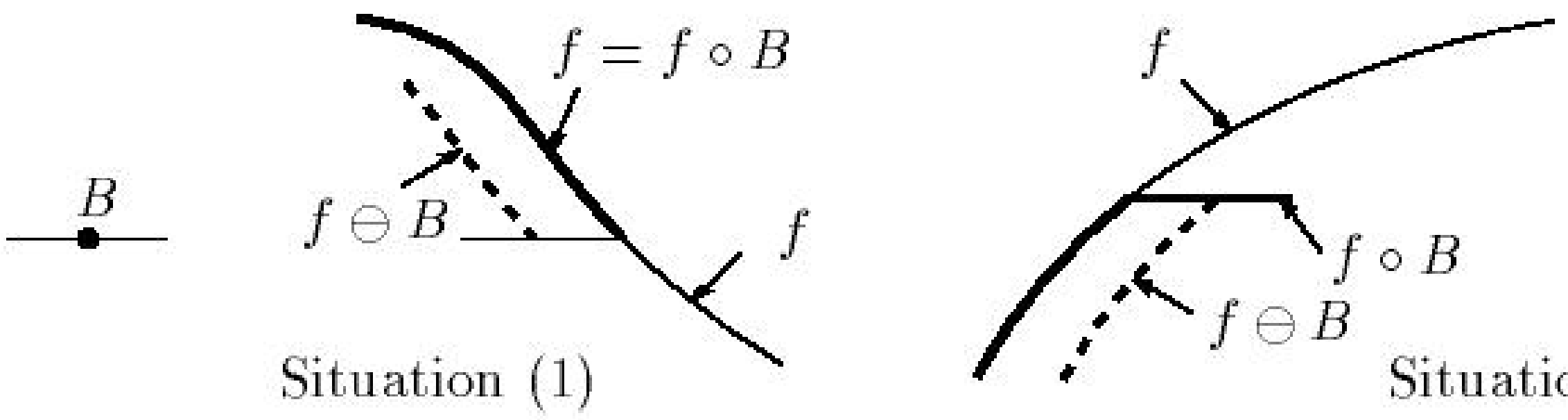

Situation (2) 
Computation time

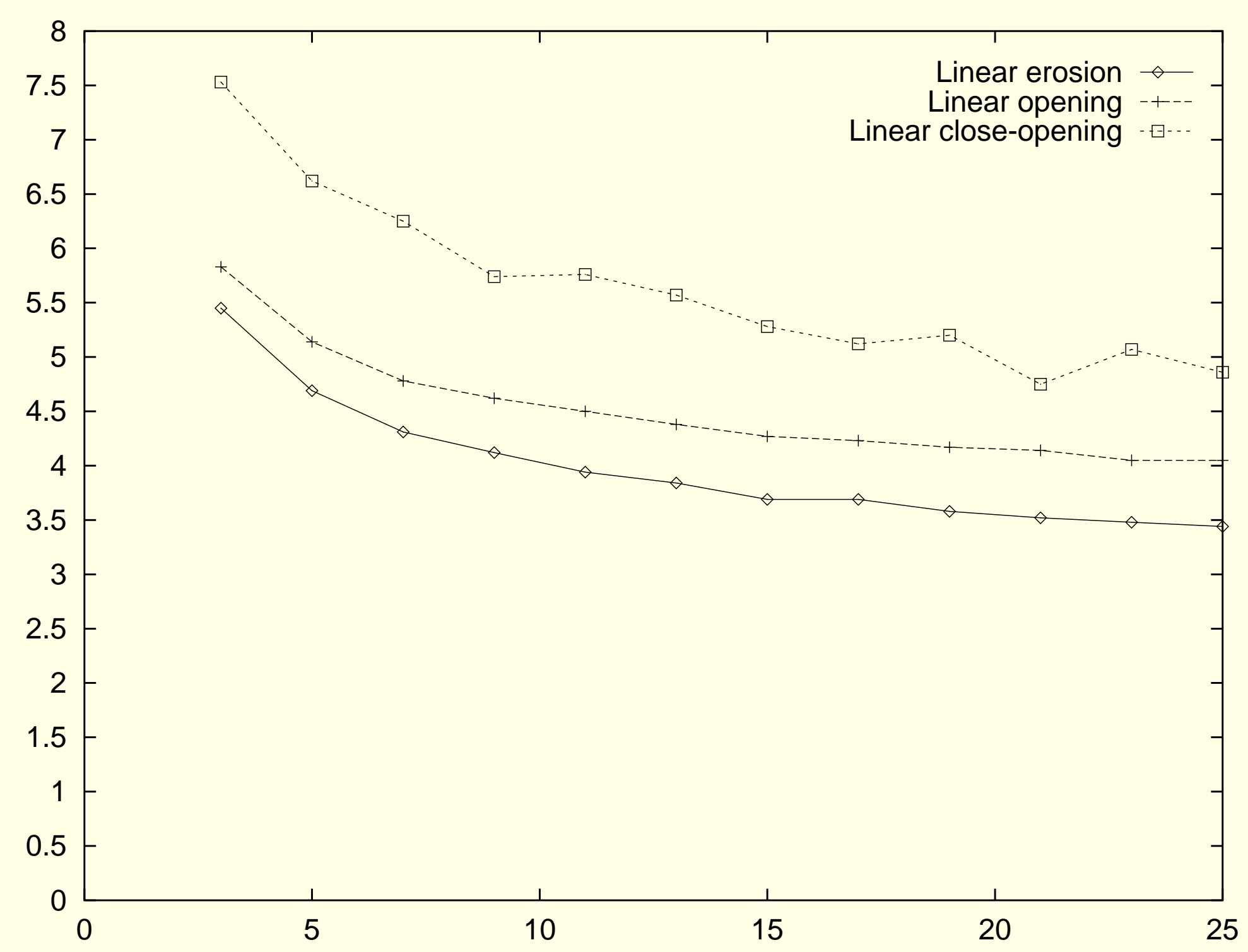


There are methods for the direct computation of linear openings (also applicable to functions)

$$
X \circ(n H \oplus m V) \neq(X \circ n H) \circ m V
$$

But direct linear opening algorithms can still help reducing the computation times as

$$
\begin{aligned}
X \circ(n H \oplus m V) & =(((X \ominus n H) \ominus m V) \oplus n H) \oplus m V \\
& =(((X \ominus n H) \ominus m V) \oplus m V) \oplus n H \\
& =((X \ominus n H) \circ m V) \oplus n H
\end{aligned}
$$




\section{First family of algorithms: 2D scan-based algorithms}

2 phases:

1. Scan the input image and build two matrices.

2. Fill the output image with thresholded values.

Our algorithms are similar to algorithms that use thresholded distance maps. 


\section{Description of the first phase}

The first matrix, denoted $H O R[i, j]$ or $H O R$, is filled with the distance of each pixel contained in an object to the right border of that object.

\begin{tabular}{llllllllllllllllllll}
\multicolumn{11}{c|}{ Original } & HOge \\
1 & 1 & 1 & 1 & 1 & 0 & 0 & 0 & 1 & 0 & 5 & 4 & 3 & 2 & 1 & 0 & 0 & 0 & 1 & 0 \\
1 & 1 & 1 & 1 & 1 & 1 & 0 & 1 & 0 & 0 & 6 & 5 & 4 & 3 & 2 & 1 & 0 & 1 & 0 & 0 \\
0 & 1 & 1 & 1 & 1 & 1 & 1 & 1 & 0 & 0 & 0 & 7 & 6 & 5 & 4 & 3 & 2 & 1 & 0 & 0 \\
1 & 1 & 1 & 1 & 1 & 1 & 1 & 1 & 1 & 0 & 9 & 8 & 7 & 6 & 5 & 4 & 3 & 2 & 1 & 0 \\
0 & 1 & 1 & 1 & 1 & 1 & 1 & 1 & 1 & 0 & 0 & 8 & 7 & 6 & 5 & 4 & 3 & 2 & 1 & 0 \\
0 & 1 & 0 & 0 & 1 & 1 & 1 & 1 & 1 & 1 & 0 & 1 & 0 & 0 & 6 & 5 & 4 & 3 & 2 & 1 \\
1 & 1 & 1 & 0 & 1 & 1 & 1 & 1 & 1 & 1 & 3 & 2 & 1 & 0 & 6 & 5 & 4 & 3 & 2 & 1 \\
1 & 1 & 1 & 1 & 0 & 1 & 1 & 1 & 1 & 1 & 4 & 3 & 2 & 1 & 0 & 5 & 4 & 3 & 2 & 1
\end{tabular}




\section{Building the second matrix: program fragment}

$V E R[i, j]$ is the length of the vertical segment of HOR that has all its value larger or equal to $H O R[i, j]$.

for all $i, j$ do

if $\operatorname{HOR}[i, j] \neq 0$ then

length $\leftarrow 1$

I* Scan to the top */

$k \leftarrow j-1$

while $H O R[i, k] \geq H O R[i, j]$ do

length $\leftarrow$ length +1

$k \leftarrow k-1$

end while

I* Scan to the bottom * /

$k \leftarrow j+1$

while $\operatorname{HOR}[i, k] \geq \operatorname{HOR}[i, j]$ do

length $\leftarrow$ length +1

$k \leftarrow k+1$

end while

$V E R[i, j] \leftarrow$ length

else

$V E R[i, j] \leftarrow 0$

end if 


\section{First phase: final result}

$\begin{array}{llllllllllllllllllllllllllllll}\text { Original image } & 110 \\ 1 & 1 & 1 & 1 & 1 & 0 & 0 & 0 & 1 & 0 & 5 & 4 & 3 & 2 & 1 & 0 & 0 & 0 & 1 & 0 & 2 & 5 & 5 & 5 & 7 & 0 & 0 & 0 & 1 & 0 \\ 1 & 1 & 1 & 1 & 1 & 1 & 0 & 1 & 0 & 0 & 6 & 5 & 4 & 3 & 2 & 1 & 0 & 1 & 0 & 0 & 1 & 4 & 4 & 4 & 6 & 7 & 0 & 7 & 0 & 0 \\ 0 & 1 & 1 & 1 & 1 & 1 & 1 & 1 & 0 & 0 & 0 & 7 & 6 & 5 & 4 & 3 & 2 & 1 & 0 & 0 & 0 & 3 & 3 & 3 & 5 & 6 & 6 & 7 & 0 & 0 \\ 1 & 1 & 1 & 1 & 1 & 1 & 1 & 1 & 1 & 0 & 9 & 8 & 7 & 6 & 5 & 4 & 3 & 2 & 1 & 0 & 1 & 2 & 2 & 2 & 4 & 5 & 5 & 5 & 5 & 0 \\ 0 & 1 & 1 & 1 & 1 & 1 & 1 & 1 & 1 & 0 & 0 & 8 & 7 & 6 & 5 & 4 & 3 & 2 & 1 & 0 & 0 & 2 & 2 & 2 & 4 & 5 & 5 & 5 & 5 & 0 \\ 0 & 1 & 0 & 0 & 1 & 1 & 1 & 1 & 1 & 1 & 0 & 1 & 0 & 0 & 6 & 5 & 4 & 3 & 2 & 1 & 0 & 8 & 0 & 0 & 2 & 3 & 3 & 3 & 3 & 3 \\ 1 & 1 & 1 & 0 & 1 & 1 & 1 & 1 & 1 & 1 & 3 & 2 & 1 & 0 & 6 & 5 & 4 & 3 & 2 & 1 & 2 & 2 & 2 & 0 & 2 & 3 & 3 & 3 & 3 & 3 \\ 1 & 1 & 1 & 1 & 0 & 1 & 1 & 1 & 1 & 1 & 4 & 3 & 2 & 1 & 0 & 5 & 4 & 3 & 2 & 1 & 1 & 1 & 1 & 1 & 0 & 3 & 3 & 3 & 3 & 3\end{array}$

Figure 2: A binary object and its corresponding two matrices $H O R$ and $V E R$.

All rectangles included in $X$ are mentioned in $H O R$ and $V E R$

$\underline{\text { but }}$

there is some redundancy 
The second phase (shown for $B=4 H \oplus 4 V$ ) 
1. Selection of positions where $\operatorname{HOR}[i, j] \geq 4$ and $\operatorname{VER}[i, j] \geq 4$

\section{Original image}

$\begin{array}{llllllllll}1 & 1 & 1 & 1 & 1 & 0 & 0 & 0 & 1 & 0\end{array}$

$\begin{array}{llllllllll}1 & 1 & 1 & 1 & 1 & 1 & 0 & 1 & 0 & 0\end{array}$

$\begin{array}{llllllllll}0 & 1 & 1 & 1 & 1 & 1 & 1 & 1 & 0 & 0\end{array}$

$\begin{array}{llllllllll}1 & 1 & 1 & 1 & 1 & 1 & 1 & 1 & 1 & 0\end{array}$

$\begin{array}{llllllllll}0 & 1 & 1 & 1 & 1 & 1 & 1 & 1 & 1 & 0\end{array}$

$\begin{array}{llllllllll}0 & 1 & 0 & 0 & 1 & 1 & 1 & 1 & 1 & 1\end{array}$

$\begin{array}{llllllllll}1 & 1 & 1 & 0 & 1 & 1 & 1 & 1 & 1 & 1\end{array}$

$\begin{array}{llllllllll}1 & 1 & 1 & 1 & 0 & 1 & 1 & 1 & 1 & 1\end{array}$
HOR

5 (4) $3 \begin{array}{lllllll}2 & 1 & 0 & 0 & 0 & 1 & 0\end{array}$

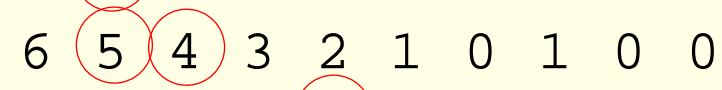

$\begin{array}{lllllllllllllllll}0 & 7 & 6 & 5 & 4 & 3 & 2 & 1 & 0 & 0\end{array}$

$\begin{array}{lllllllllll}9 & 8 & 7 & 6 & 5 & 4 & 3 & 2 & 1 & 0\end{array}$

$\begin{array}{llllllllll}0 & 8 & 7 & 6 & 5 & 4 & 3 & 2 & 1 & 0\end{array}$

$\begin{array}{llllllllll}0 & 1 & 0 & 0 & 6 & 5 & 4 & 3 & 2 & 1\end{array}$

$\begin{array}{llllllllll}3 & 2 & 1 & 0 & 6 & 5 & 4 & 3 & 2 & 1\end{array}$

$\begin{array}{llllllllll}4 & 3 & 2 & 1 & 0 & 5 & 4 & 3 & 2 & 1\end{array}$
VER

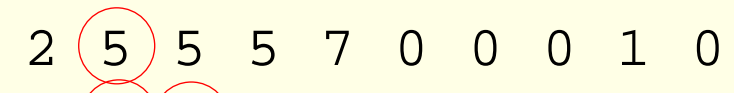

1 (4) 4 4 $46 \begin{array}{llllll} & 6 & 0 & 7 & 0 & 0\end{array}$

$\begin{array}{llllllllll}0 & 3 & 3 & 3 & 5 & 6 & 6 & 7 & 0 & 0\end{array}$

$\begin{array}{llllllllll}1 & 2 & 2 & 2 & 4 & 5 & 5 & 5 & 5 & 0\end{array}$

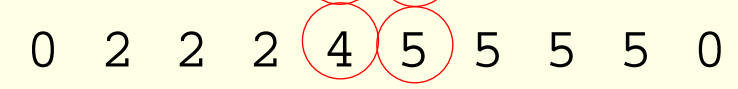

$\begin{array}{llllllllll}0 & 8 & 0 & 0 & 2 & 3 & 3 & 3 & 3 & 3\end{array}$

$\begin{array}{llllllllll}2 & 2 & 2 & 0 & 2 & 3 & 3 & 3 & 3 & 3\end{array}$

$\begin{array}{llllllllll}1 & 1 & 1 & 1 & 0 & 3 & 3 & 3 & 3 & 3\end{array}$

2. Fill the first column with run-length values

3. Fill the output image

\section{original image}

$\begin{array}{llllllllll}1 & 1 & 1 & 1 & 1 & 0 & 0 & 0 & 1 & 0\end{array}$

$\begin{array}{llllllllll}1 & 1 & 1 & 1 & 1 & 1 & 0 & 1 & 0 & 0\end{array}$

$\begin{array}{llllllllll}0 & 1 & 1 & 1 & 1 & 1 & 1 & 1 & 0 & 0\end{array}$

$\begin{array}{llllllllll}1 & 1 & 1 & 1 & 1 & 1 & 1 & 1 & 1 & 0\end{array}$

$\begin{array}{llllllllll}0 & 1 & 1 & 1 & 1 & 1 & 1 & 1 & 1 & 0\end{array}$

$\begin{array}{llllllllll}0 & 1 & 0 & 0 & 1 & 1 & 1 & 1 & 1 & 1\end{array}$

$\begin{array}{llllllllll}1 & 1 & 1 & 0 & 1 & 1 & 1 & 1 & 1 & 1\end{array}$

$\begin{array}{llllllllll}1 & 1 & 1 & 1 & 0 & 1 & 1 & 1 & 1 & 1\end{array}$
INTERMEDIATE RESULT

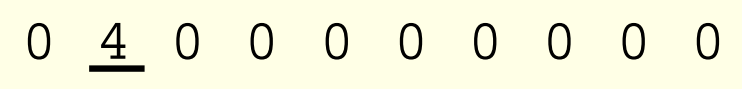

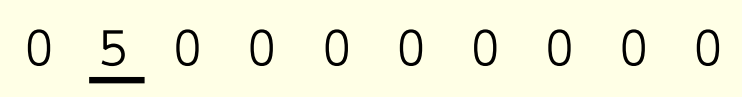

$\begin{array}{llllllllll}0 & 5 & 0 & 0 & \underline{4} & 0 & 0 & 0 & 0 & 0\end{array}$

$\begin{array}{llllllllll}0 & 5 & 0 & 0 & \underline{5} & 4 & 0 & 0 & 0 & 0\end{array}$

$\begin{array}{llllllllll}0 & 5 & 0 & 0 & 5 & 4 & 0 & 0 & 0 & 0\end{array}$

$\begin{array}{llllllllll}0 & 0 & 0 & 0 & 5 & 4 & 0 & 0 & 0 & 0\end{array}$

$\begin{array}{llllllllll}0 & 0 & 0 & 0 & 5 & 4 & 0 & 0 & 0 & 0\end{array}$

$\begin{array}{llllllllll}0 & 0 & 0 & 0 & 0 & 4 & 0 & 0 & 0 & 0\end{array}$
OUT

$\begin{array}{llllllllll}0 & 1 & 1 & 1 & 1 & 0 & 0 & 0 & 0 & 0\end{array}$

$\begin{array}{llllllllll}0 & 1 & 1 & 1 & 1 & 1 & 0 & 0 & 0 & 0\end{array}$

$\begin{array}{llllllllll}0 & 1 & 1 & 1 & 1 & 1 & 1 & 1 & 0 & 0\end{array}$

$\begin{array}{llllllllll}0 & 1 & 1 & 1 & 1 & 1 & 1 & 1 & 1 & 0\end{array}$

$\begin{array}{llllllllll}0 & 1 & 1 & 1 & 1 & 1 & 1 & 1 & 1 & 0\end{array}$

$\begin{array}{llllllllll}0 & 0 & 0 & 0 & 1 & 1 & 1 & 1 & 1 & 0\end{array}$

$\begin{array}{llllllllll}0 & 0 & 0 & 0 & 1 & 1 & 1 & 1 & 1 & 0\end{array}$

$\begin{array}{llllllllll}0 & 0 & 0 & 0 & 0 & 1 & 1 & 1 & 1 & 0\end{array}$ 


\section{Implementation issues: optimizations}

- Better use of hardware and software characteristics.

- Because of to the linear structure of memory blocks, accesses along a row in a 2-D array are faster than accesses along a column.

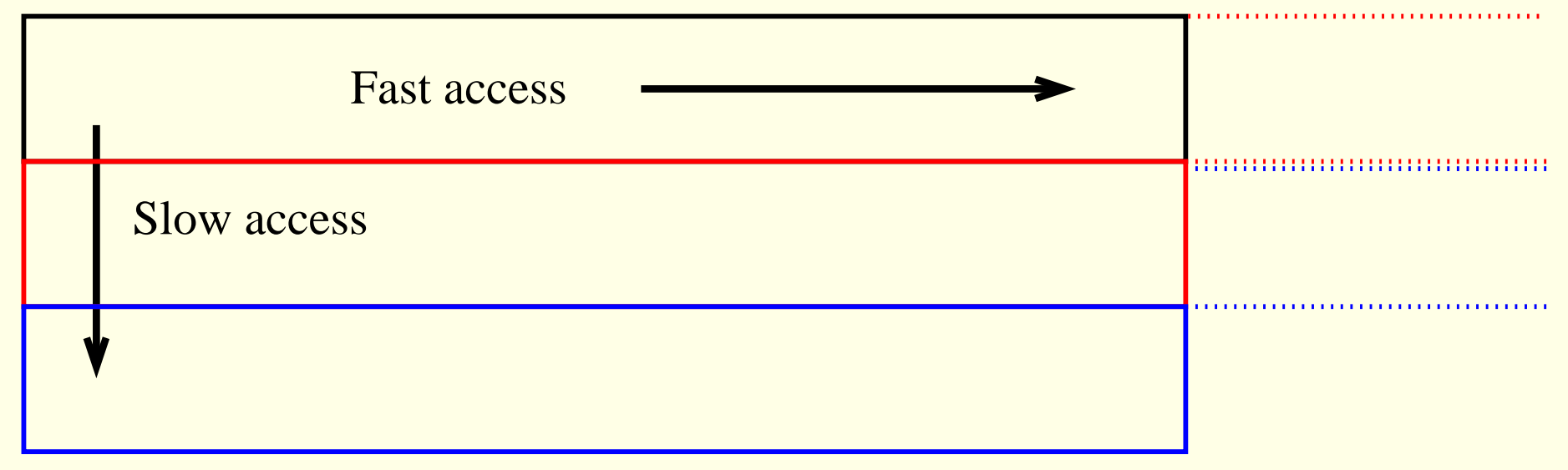

- HOR is transposed before being stored. 


\section{Implementation issues: optimizations}

- Better use of hardware and software characteristics.

- Because of to the linear structure of memory blocks, accesses along a row in a 2-D array are faster than accesses along a column.

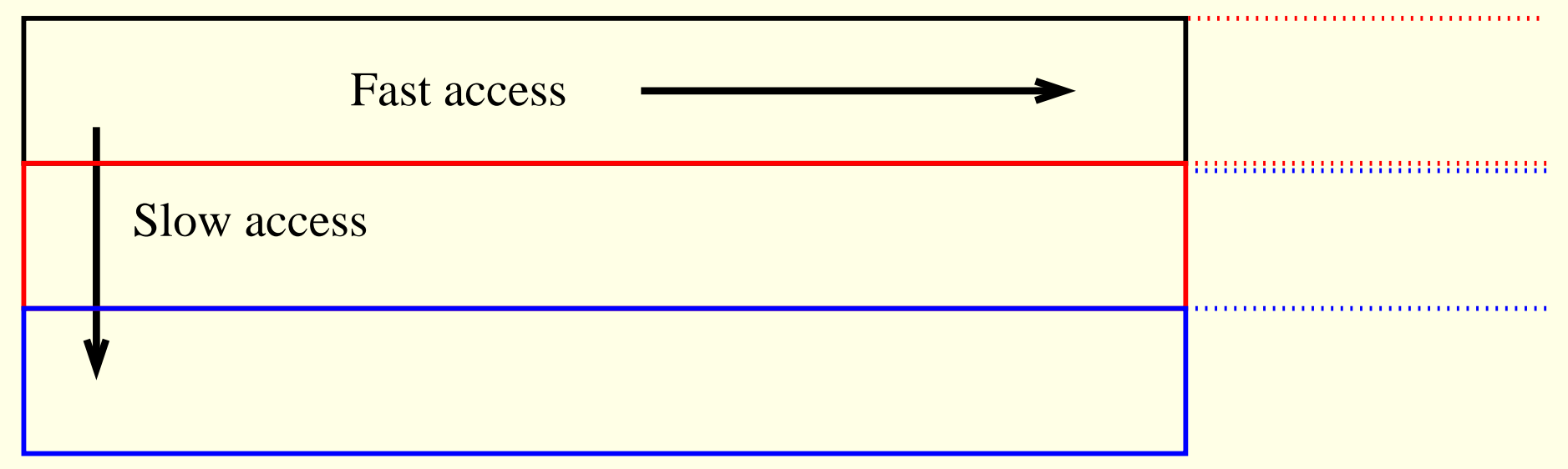

- HOR is transposed before being stored.

- Avoid some computation redundancy.

- If $H O R[i, j]=H O R[i, j-1]$ then $\operatorname{VER}[i, j]=\operatorname{VER}[i, j-1]$.

All these optimizations were included in the implementations. 


\section{Generalization of the 2D scan-based algorithm to label images}

$$
(B=3 H \oplus 3 V)
$$

LABEL IMAGE

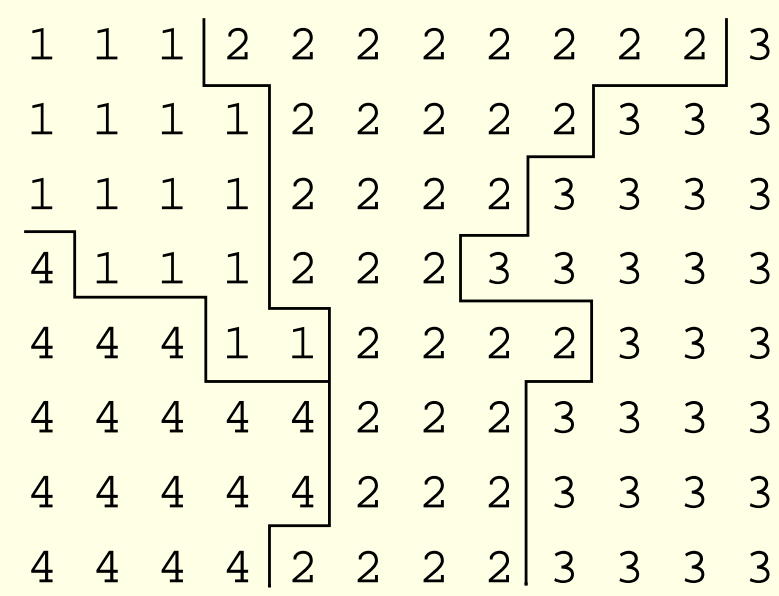

HOR

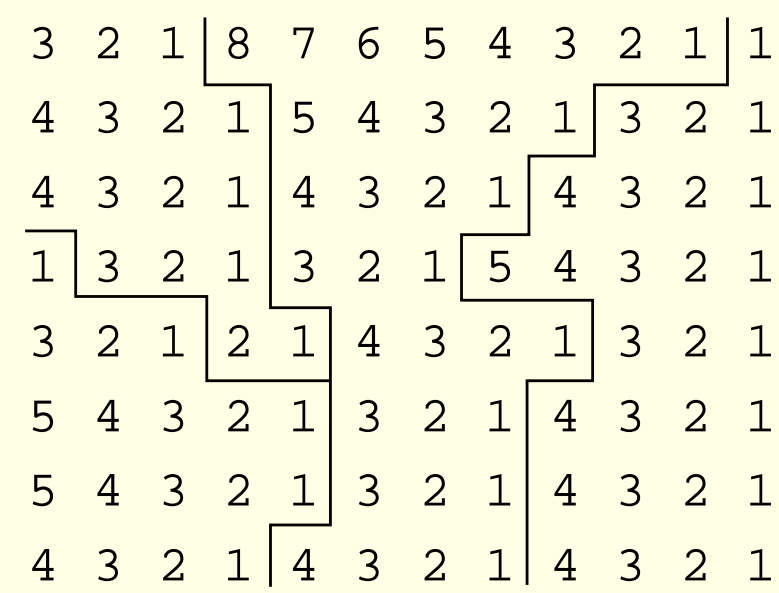

VER

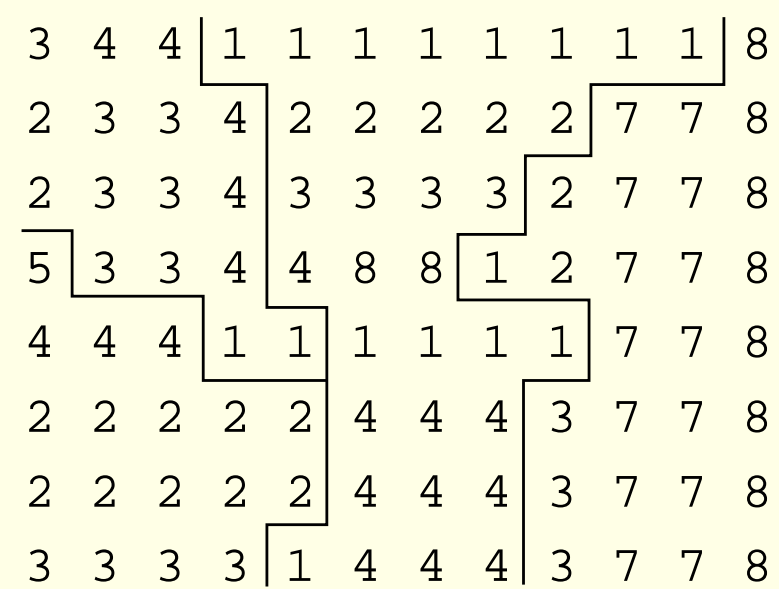

OUT

\begin{tabular}{lll|l:llll:lll|l}
1 & 1 & 1 & 0 & 2 & 2 & 2 & 2 & 0 & 0 & 0 & 0 \\
1 & 1 & 1 & 1 & 2 & 2 & 2 & 2 & 0 & 3 & 3 & 3 \\
1 & 1 & 1 & 1 & 2 & 2 & 2 & 2 & 0 & 3 & 3 & 3 \\
\hline 0 & 1 & 1 & 1 & 2 & 2 & 2 & 0 & 0 & 3 & 3 & 3 \\
\hdashline 4 & 4 & 4 & 0 & 0 & 2 & 2 & 2 & 0 & 3 & 3 & 3 \\
4 & 4 & 4 & 0 & 0 & 2 & 2 & 2 & 3 & 3 & 3 & 3 \\
4 & 4 & 4 & 0 & 0 & 2 & 2 & 2 & 3 & 3 & 3 & 3 \\
4 & 4 & 4 & 0 & 0 & 2 & 2 & 2 & 3 & 3 & 3 & 3
\end{tabular}




\section{Second family of algorithms: list-based algorithms}

Principle: build a list of rectangles $L=R_{0}, \ldots, R_{L}$ such that

- $\bigcup R_{i}=X$

- all rectangles of $\mathcal{L}$ are maximum in size

- the list does not contain any redundant rectangle

1000 lines of $C$ code! 


\section{Description}

$$
\begin{aligned}
& \text { Original image } \\
& \begin{array}{llllllllll}
1 & 1 & 1 & 1 & 1 & 0 & 0 & 0 & 1 & 0
\end{array}
\end{aligned}
$$

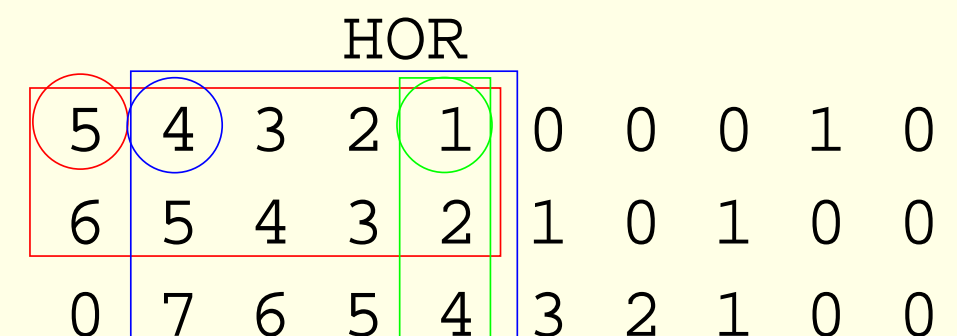

$$
\begin{aligned}
& \begin{array}{llllllllll}
1 & 1 & 1 & 1 & 1 & 1 & 0 & 1 & 0 & 0
\end{array} \\
& \begin{array}{llllllllll}
0 & 1 & 1 & 1 & 1 & 1 & 1 & 1 & 0 & 0
\end{array} \\
& \begin{array}{lllll|lllll}
9 & 8 & 7 & 6 & 5 & 4 & 3 & 2 & 1 & 0
\end{array} \\
& \begin{array}{llllllllll}
1 & 1 & 1 & 1 & 1 & 1 & 1 & 1 & 1 & 0
\end{array} \\
& \begin{array}{lllll|lllll}
0 & 8 & 7 & 6 & 5 & 4 & 3 & 2 & 1 & 0
\end{array} \\
& \begin{array}{llllllllll}
0 & 1 & 1 & 1 & 1 & 1 & 1 & 1 & 1 & 0 \\
0 & 1 & 1 & 1 & 1 & 1 & 1
\end{array} \\
& \begin{array}{llllllllll}
0 & 1 & 0 & 0 & 6 & 5 & 4 & 3 & 2 & 1
\end{array}
\end{aligned}
$$

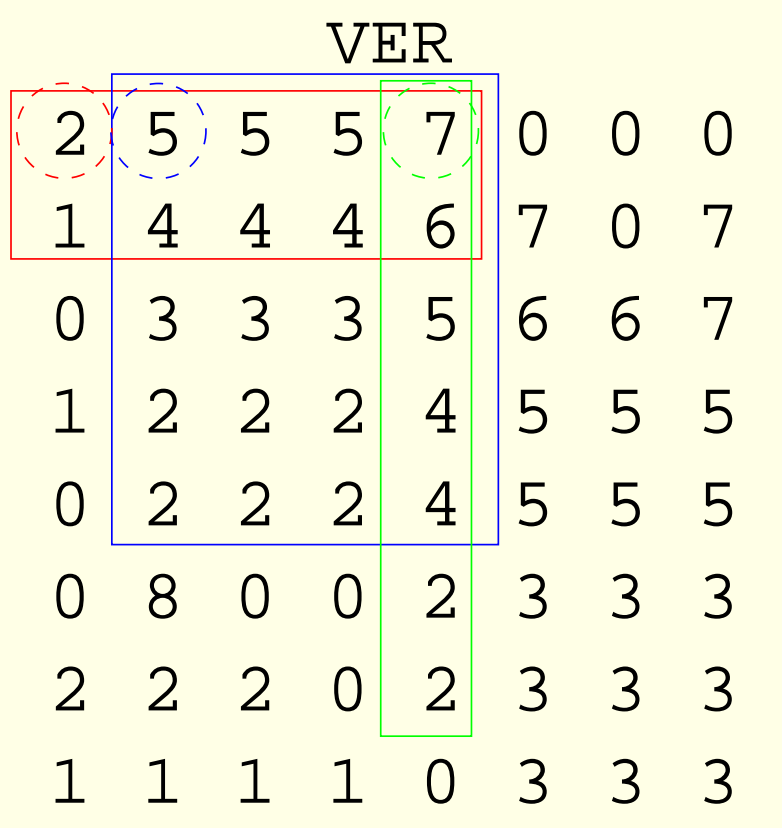

$$
\begin{aligned}
& \begin{array}{lll}
(i, j)=(0,0) & \text { Width }=5 & \text { Height }=2 \\
(i, j)=(0,0) & \text { Width }=4 & \text { Height }=5 \\
(i, j)=(0,0) & \text { Width }=1 & \text { Height }=7
\end{array} \\
& \begin{array}{llllllllll}
1 & 1 & 1 & 0 & 1 & 1 & 1 & 1 & 1 & 1
\end{array} \\
& \begin{array}{lllllllllll}
3 & 2 & 1 & 0 & 6 & 5 & 4 & 3 & 2 & 1
\end{array} \\
& \begin{array}{llllllllll}
1 & 1 & 1 & 1 & 0 & 1 & 1 & 1 & 1 & 1
\end{array} \\
& \begin{array}{llllllllll}
4 & 3 & 2 & 1 & 0 & 5 & 4 & 3 & 2 & 1
\end{array}
\end{aligned}
$$

Basically, $V E R$ is replaced by a list.

21 non-redundant rectangles. 


\section{Problem of multiple intersections}

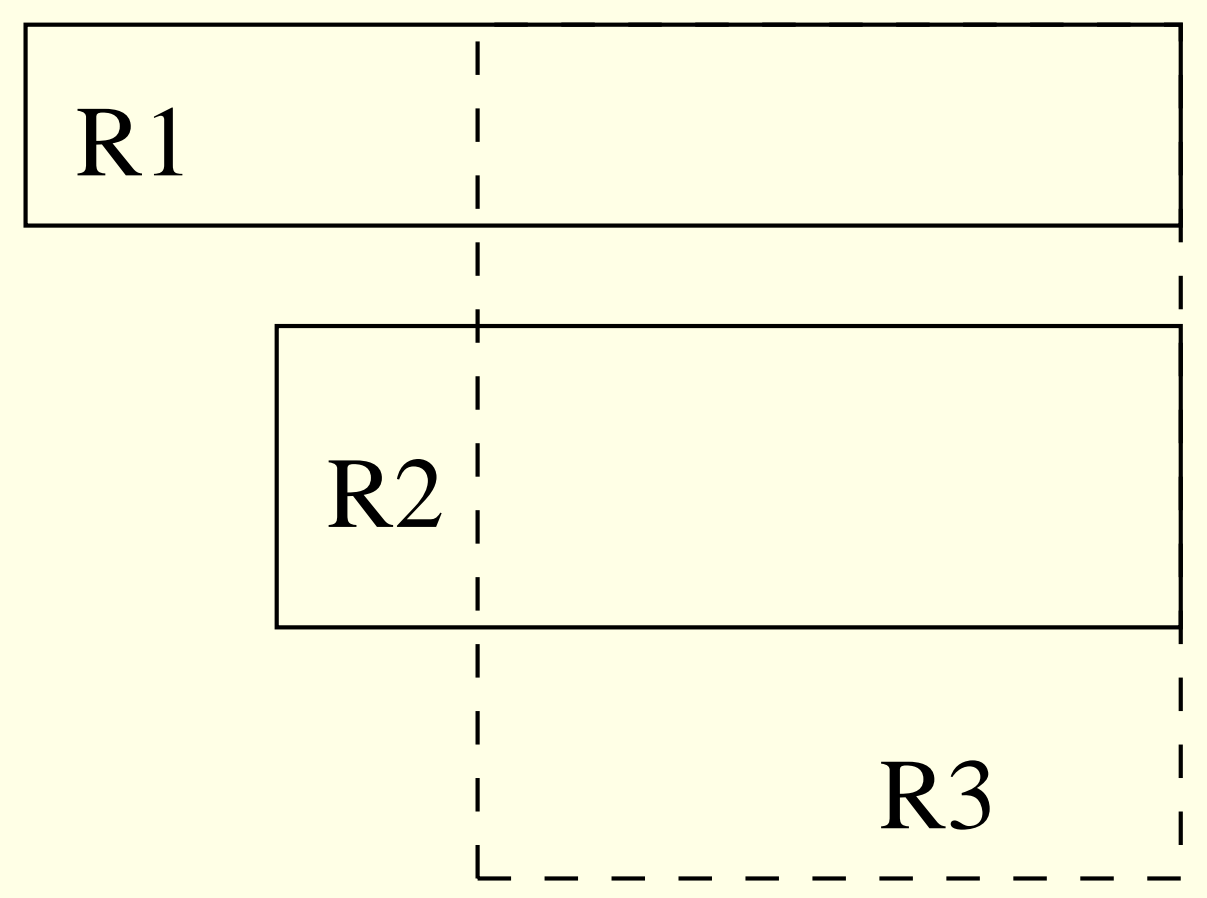

Two solutions:

1. Avoid rewriting pixels in $O U T$

2. Stores all the intersections between elements of $\mathcal{L}$.

A thorough examination of all the intersections leads to a variant (called area specific) for which no output pixel is written twice. 


\section{Computation times of 2D scan and list-based algorithms}

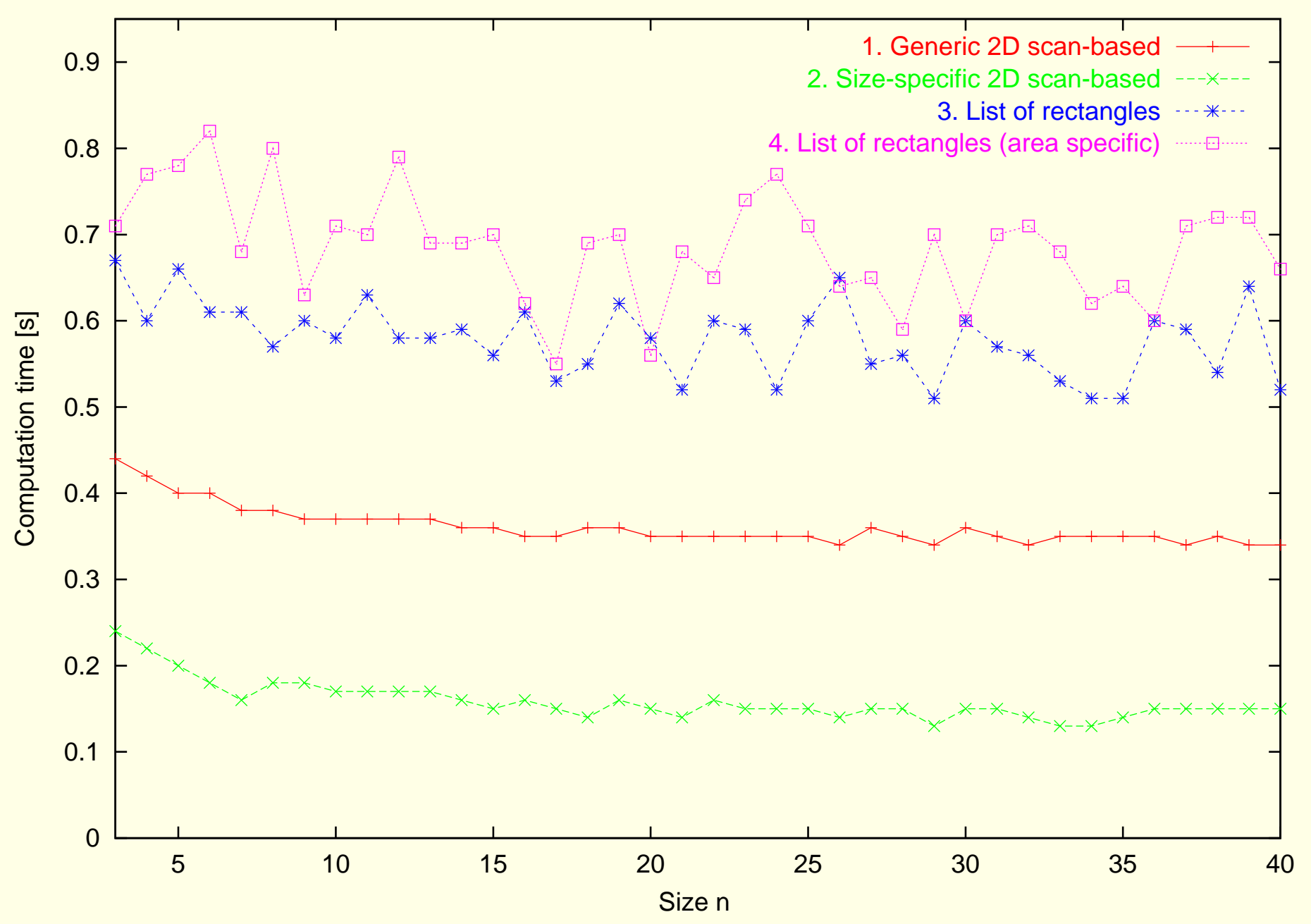


- The respective contributions of the first and second phase to the total amount of time are about $85 \%-15 \%$. 


\section{Comparison with other algorithms}

- All algorithms have been reimplemented and optimized for the case of binary images.

- For the particular case of VAN HERK's algorithm (often used as a benchmark), we transposed the intermediate matrix twice, after $X \ominus n H$ and $((X \ominus n H) \ominus m V) \oplus$ $m V$, to achieve a fair comparison with our algorithms. 


\section{Computation times of several algorithms for binary openings}




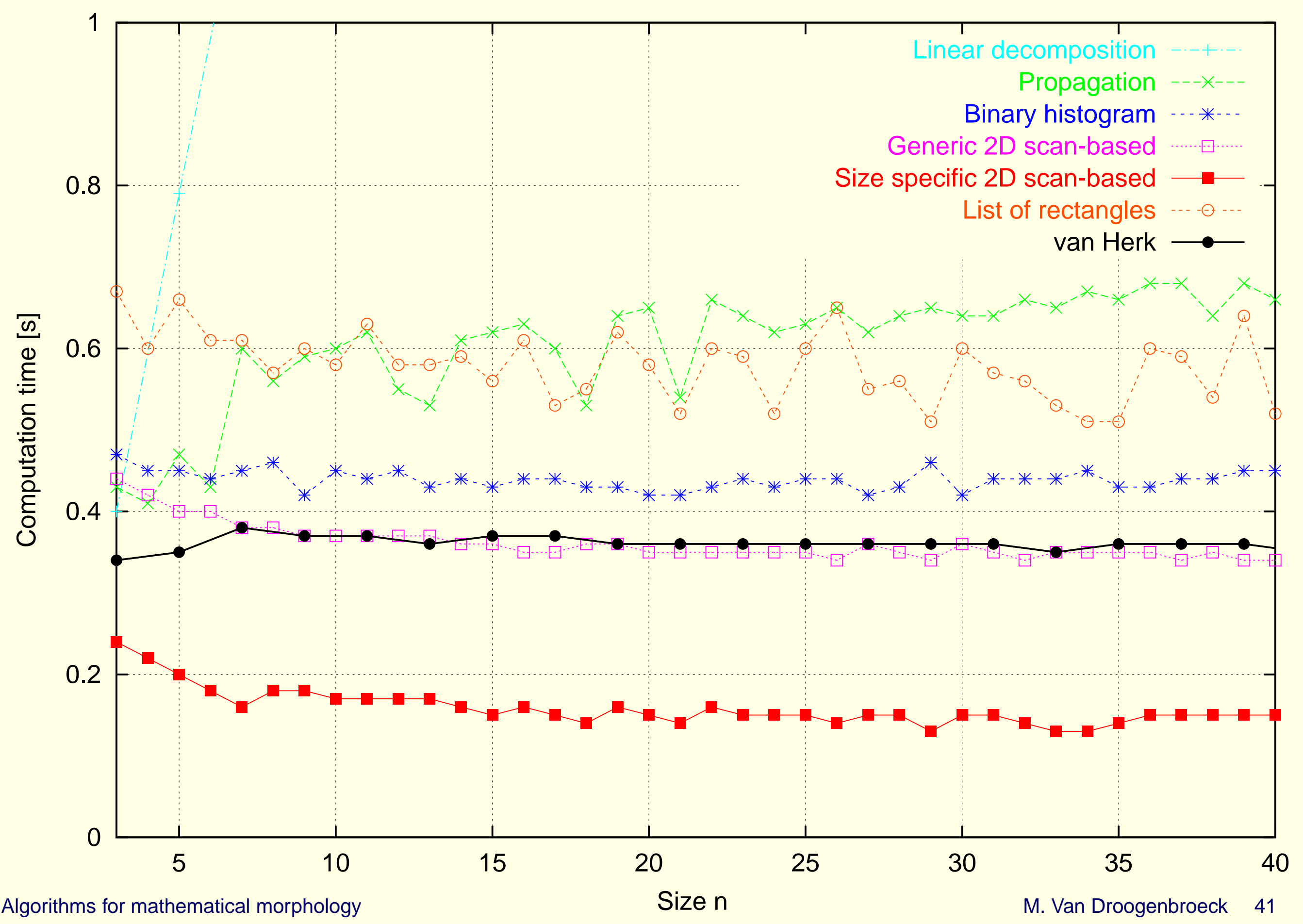




\section{Comparison of algorithms that do not depend on the size of the structuring element}

\begin{tabular}{lcccc}
\hline & Histogram & van Herk & Scan-based & List-based \\
\hline Program complexity & Very low & Low & Medium & High \\
Relative computation speed & Fast & Fast & Very fast & Slow \\
Allow multiple computations & No & No & Yes & Yes \\
Dependent on the content & Slightly & No & Yes & Yes \\
\hline
\end{tabular}




\section{Granulometries}

\section{VINCENT (2000). Granulometries and Opening Trees.}

